\title{
The Role of Telemedicine in Child and Adolescent Healthcare in India
}

\author{
Preeti M. Galagali ${ }^{1}\left[\right.$. Sreyoshi Ghosh ${ }^{2} \cdot$ Hemant Bhargav $^{3}$ \\ Accepted: 14 September 2021 / Published online: 8 October 2021 \\ (c) The Author(s), under exclusive licence to Springer Science+Business Media, LLC, part of Springer Nature 2021
}

\begin{abstract}
Purpose of Review We highlight the evolution and use of telemedicine in child and adolescent healthcare in India, in pre and post pandemic eras.

Recent Findings The latest research endorses telemedicine as a successful strategy in resource-limited settings to provide accessible and equitable healthcare.

Summary Telemedicine was initiated in India in 2001. The pandemic restrictions resulted in an increase in its use. The national telemedicine and telepsychiatry guidelines facilitated and enabled its widespread use. Telehealth was used by private and public health establishments, in urban, rural, and remote areas. It was used for triaging cases; managing and monitoring COVID patients in home isolation, in non-COVID medical and psychiatric care, and follow-up; continuing medical education; and health promotion strategies like teleyoga. The shortcomings included inability to provide privacy and confidentiality, perform physical examination, and provide emergency care. Most patients and their caregivers expressed satisfaction with telehealth services. Telemedicine is likely to become an integral part of healthcare services in the post pandemic era.
\end{abstract}

Keywords Telemedicine $\cdot$ Telepsychiatry $\cdot$ Teleyoga $\cdot$ India $\cdot$ Pandemic $\cdot$ Children and adolescents

\section{Introduction}

India has the second largest case load of COVID-19 cases in the world, with 32,512,366 cases until 25 August 2021 [1]. India is a large country with a population of 1.3 billion people and limited economic and human resources. The current doctor/patient ratio in India is 0.62:1000 compared to that recommended by the WHO as 1:1000. Seventy-five percent of the total doctors in India practice

This article is part of the Topical Collection on Technology and Pediatrics

Preeti M. Galagali

drpgalagali@gmail.com

1 Bengaluru Adolescent Care and Counselling Centre, No. 528, 10th Main Ram Mandir Road, 2nd Block Rajajinagar, Bengaluru, Karnataka 560079, India

2 National Institute of Mental Health and Neurosciences, Bengaluru, Karnataka, India

3 Department of Integrative Medicine, NIMHANS, Bengaluru, India in urban areas where only $31 \%$ of the population live

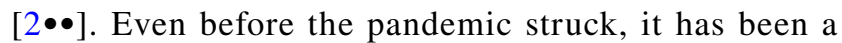
challenge to deliver optimal healthcare to India's large population, due to an overburdened healthcare system. As an additional healthcare delivery approach, telemedicine has revolutionized medical services in India during the pandemic.

Currently, $45 \%$ of the population has internet connection, and $79 \%$ has a mobile phone connection [3]. Availability of economical internet connectivity further increased the use of telehealth services. Through telemedicine, health professionals were able to reach the population living in remote areas of the country.

India reported its first case of COVID on 31 January 2020, and a lockdown was declared on 24 March 2020. During the lockdowns, the focus of healthcare establishments was on the care of COVID-affected patients. There was closure of the outpatient services. In addition, due to travel restrictions, routine healthcare services and follow-up care of patients with chronic medical, mental,

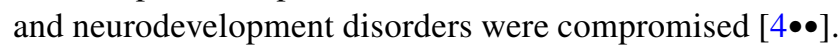
In response to the healthcare crisis precipitated by the pandemic, India released its first national telemedicine 
guidelines on 25 March 2020. With the aid of telemedicine, healthcare professionals and community frontline workers could triage paediatric patients, refer them to emergency services, treat minor illnesses, order investigations, receive reports, conduct follow-up consultations, impart health education regarding COVID appropriate behaviour, conduct research, and access continuing medical education (CME) programs and clinical protocols. Use of telemedicine reduced the exposure of healthcare professionals and non-COVID patients to COVID infection by decreasing the footfall in hospitals.

We review the growth of telemedicine and psychiatry in India, focus on its role during the pandemic in child and adolescent medical and mental healthcare, and discuss a way forward for its integration in routine medical care in future. In a resource-limited settings typical of many low- and middle-income (LMIC) countries, judicious use of telemedicine could enable equitable healthcare services for all.

\section{Definition}

Telemedicine has been defined by the World Health Organization (WHO) as the delivery of healthcare services, where distance is a critical factor, by healthcare professionals using information and communication technologies for the exchange of valid information for the diagnosis, treatment, and prevention of disease and injuries; for research and evaluation; and for the continuing education of healthcare providers, all in the interest of advancing the health of individuals and their communities. Disease management, health promotion, research, and medical education fall under the purview of telemedicine. Telemedicine is an important component of the digital health strategy 2020-2024 adopted by the WHO to facilitate equitable and universal access to health services, personalised care, and preparedness to deal with emergencies $[5 \bullet]$.

\section{Evolution of Telemedicine in India}

In 2001, India launched its first telemedicine program named the Telemedicine Pilot Project, led by the Indian Space Research Organisation that linked a village hospital to a specialized urban heart care centre in the state of Tamil Nadu. In 2002, there was a report of triaging neonatal cases in rural areas via email [6]. In 2004, village resource centres were set up in 500 villages to provide telemedicine services through ASHA workers [2••]. In 2005, a National Telemedicine Taskforce was established by the Government of India that initiated various programs like the Integrated Disease Surveillance Project, the National Cancer Network, the National Rural Telemedicine Network, the Digital Medical Library Network, and the National Medical College

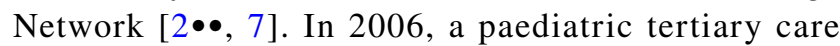
centre in India established a teleconsultation service to reach out to underprivileged children in rural areas that resulted in considerable monetary savings for the families [8]. Telemedicine has also been used to deliver healthcare during mass religious gatherings like Maha Kumbh Mela. In 2012, mobile technologies were used by frontline health workers to promote breast feeding and kangaroo mother care and improve complementary feeding practices [9]. For over a decade, mHealth has been in use in the field of neonatology in India for dissemination of evidence-based guidelines for neonatal care, collection of data, and research purposes [10]. Recently, 45 rural health centres were connected to 15 specialized hospitals for eye, cardiac, and cancer care services via telemedicine. In 2014, India launched the national health portal. Through this portal, a free telemedicine service called eSanjeevani can be accessed. The health portal also provides health education, details of health facilities, hospitals, blood transfusion centres, mobile apps, and personalized health records [11]. It was only during the pandemic that India adopted telemedicine universally and published the official guidelines for its use.

\section{Telemedicine Guidelines}

The Indian telemedicine guidelines propose that the first consultation must be conducted face to face and hence must be a video consultation [12]. Follow-up consultations may be conducted telephonically or over text or email as well, for a time duration of up to 6 months after which another video consultation is mandated. A summary of the Indian telemedicine guidelines is given in Box 1. These guidelines have enabled patients living in rural and remote areas of the country to access healthcare by a registered medical practitioner. Through telehealth, a community health worker can now legally seek help from specialists from any part of the country for treating patients in villages. Telehealth has literally brought health services to the doorstep of all citizens of India. The guidelines clearly state that telemedicine should not be used for invasive or surgical procedures or for treating patients outside the jurisdiction of India. 
Box 1 Summary of Indian telemedicine guidelines for registered medical practitioners (RMP).

- The teleconsultation can be conducted via the audio, text, or video modes

- Asynchronous or synchronous methods can be adopted

- It should be adopted for only non-emergency cases as a first consult or for follow-up or for health education or counselling purposes

- The teleconsultation can be availed by the patient, caregiver, another RMP, or a healthcare worker

- An individual above 16 years of age can give consent for teleconsultation, while the caregivers consent is required for younger age groups. The explicit consent from the caregivers should be documented and recorded

- The treatment should be written in the prescribed format. The prescription can be shared online with the patient. Records should be maintained

- Over-the-counter drugs and only the specific class of drugs listed in the guidelines can be prescribed via the telehealth services. Injectable medicines cannot be prescribed to the patients

- The medical records should be kept confidential

- RMP is not legally liable for the breach of online data due to technical errors

- All RMP should undergo a specialised training in telemedicine

\section{Telehealth for Medical Disorders in COVID Era}

Telemedicine has been widely used by primary healthcare centres, clinics, and tertiary care hospitals during the pandemic. A summary of its effective usage by paediatric hospitals and specialists is given below. Paediatric and adolescent telemental health services would be discussed in the next section.

1. Triage of COVID patients: Teleconsultation was used to triage patients of COVID and for management and follow-up of patients in home isolation [13]. Collaborations were made with experts in other countries to facilitate management of a high case load of COVID patients [14]. To restrict the exposure of health professionals to COVID, a few hospitals in India adopted software that used the telephonic triage system to give physical appointments to select patients [15]. Keeping in mind the success of this model, hospitals plan to continue using this system in the post pandemic era to decrease overcrowding of the hospital premises.

2. Ophthalmology: In the pre pandemic era, telemedicine was used to screen and triage high-risk babies for retinopathy of prematurity in rural areas [16]. During the pandemic, paediatric cases of strabismus, oculoplasty, refractive errors, stereopsis, stye, chalazion, and conjunctivitis were managed through teleophthalmology [17]. This mode was also used to follow up operated cases. Diseases of the posterior segment of the eye and those requiring slit lamp examination and tonometer were given physical appointments.

3. HIV care: Through a paediatric HIV telemedicine initiative, high-quality care was effectively provided to a rural population of children with HIV $[18,19]$. The patients were provided with expert guidance regarding anti-retroviral therapy and were given follow-up appointments. An increase in compliance to treatment and decreased deaths in the study population were observed.

4. Paediatric surgery follow-up: Telephonic calls were found to be effective in follow-up of patients who had undergone surgery just before the lockdown. Partnering with local doctors who were located near the patients' houses was helpful in providing emergency treatment [20].

5. Pulmonology: Expert group recommendations for paediatric telepulmonology services emphasized on the need for triaging before physical consultation and facilitating investigations through telemedicine [21]. Asthma follow-up and medicine refills were successfully conducted with high caregiver satisfaction [22].

6. Neurology: For neurology cases, a video consultation was the preferred mode of teleconsultation [23]. The main limitation was the inability to do a neurological physical examination. Migraine and epilepsy follow-up could be easily done via video or telephonic consultation with over $90 \%$ of the caregivers being satisfied with the telemedicine services [24, 25].

7. Neurodevelopmental follow-up: General motor assessment of high-risk infants was conducted effectively by analysing videos and interventions were planned [26].

8. Oncology: Email and telephone calls were used for non-intensive care and follow-up of paediatric oncology services. A shared care model was followed by paediatric oncologists by partnering with local healthcare facilities and guiding them regarding chemotherapy, blood product infusion, and management of complications [27].

9. Nephrology: Telehealth services via video and audio consultation were used successfully to follow up children with chronic kidney disease, and a few new cases were diagnosed with high caregiver satisfaction [28, 29].

10. Diabetes care: Telemedicine services were used for triaging, follow-up, parent education, and health education of children with diabetes mellitus [30].

The digital platform of Indian Academy of Pediatrics conducted over a thousand webinars to train paediatricians regarding latest guidelines of various child and adolescent healthcare issues including COVID-19 protocols [11]. 
Research studies are currently being conducted in India to promote resilience in the youth during the pandemic [31]. With increased use of telemedicine, a change in guidelines may be required, for example, including prescriptions for abortion services for young adults [32]. Telemedicine has been very effective in providing non-emergency medical healthcare and counselling services and for referring child and adolescent patients to appropriate healthcare establishments during the pandemic.

\section{Clinical Services at a Virtual Adolescent Clinic During COVID-19}

Bengaluru Adolescent Care and Counselling Centre is a private establishment providing comprehensive healthcare services to adolescents and young adults for 16 years. Due to the pandemic, the services were shifted to the online mode. From April 2020 to August 2021, 250 adolescents were given therapeutic and follow-up telehealth services. All patients were asked to visit the clinic for one brief physical consultation to facilitate rapport building and screening for medical disorders. The health issues that were managed through the teleconsult mode, in accordance with the Indian telemedicine guidelines, included COVID-19, acne, headache, menstrual issues, upper respiratory infections, asthma, allergic rhinitis, obesity, anaemia, excessive media usage, cyberbullying, depression, anxiety, eating disorder, conflicts with parents, anger, aggression, bereavement, and poor academic performance. Parents of the adolescents were also counselled online regarding positive parenting techniques, handling their stress, and monitoring the behaviour of their wards. The links of IAP parenting handouts and webinars were shared with them (https://iapindia.org/guidelines-forparents/). Cases of severe mental disorders like obsessive compulsive disorders, suicidal behaviour, substance use disorder, and schizophrenia were referred to the child and adolescent psychiatrist. Community outreach activities of the centre like imparting parenting training and school health education also continued over the online mode. A total of sixteen such activities were conducted. An online survey was conducted for 2000 students to screen for psychosocial and emotional issues. The results of this survey were used to design the contents of the adolescent education programs.

Though the teleconsult mode enabled the centre to deliver therapeutic and counselling services during the pandemic, it was challenging to ensure privacy and confidentiality for the individual sessions due to interference of parents and inability to arrange a secluded place for the session. During the community sessions, it was difficult to interact freely with the audience. The pros and cons of telemedicine are en-numerated in Box 2.
Box 2 Pros and cons of telemedicine.

\begin{tabular}{ll}
\hline Pros & Cons \\
\hline - Availability and easy acces- & - Technological issues: poor inter- \\
sibility of health services, & net bandwidth, digital illiteracy \\
especially in remote and rural & $\begin{array}{c}\text { - Missed/error in diagnosis due } \\
\text { to inability of doing physical }\end{array}$ \\
areas & examination \\
- Decreases footfalls in hospitals & - Ineffective counselling due to \\
- Physical distancing & poor online recognition of non- \\
- Triaging & verbal and verbal cues \\
- First aid & Prescription errors: inability to \\
- Refill medicines & understand prescription due to \\
- Follow-up of chronic medical & illiteracy \\
disorders & - Privacy, confidentiality, and \\
- Post surgical follow-up & distraction challenges \\
- Health education for individu- & - Inability to manage medicolegal \\
als and the community & cases \\
- Collaborative specialty care & Health data prone to hacking \\
with primary care physician & and misuse \\
and village health worker & and
\end{tabular}

\section{Telepsychiatry Services}

The practice of telepsychiatry typically involves either a synchronous (real-time) or an asynchronous (forwarding medical records for expert opinion) format [33]. The synchronous, two-way, interactive telecommunication format is more popular, often in video teleconferencing (VTC). This has also been the usual format for teleconsultations in tertiary mental healthcare hospitals such as the National Institute of Mental Health and Neurosciences (NIMHANS) in India, during and before the pandemic.

Even before the onset of the pandemic, between 2017 and 2020, the Telepsychiatry after care Clinics at NIMHANS, India, had provided 780 teleconsultations for 232 clients with various psychiatric disorders [34]. Another telepsychiatry project in India by Thara et al. in 2008 [35] offered services to 156 villages using a bus that was custom-built to accommodate a consultation room and pharmacy. After a teleconsultation, a prescription was advised by the psychiatrist located at the nodal centre to the telepsychiatry clinic facilitator in the bus and dispensed by the onboard pharmacy. Specifically for Indian youth, Malhotra et al. in 2016 [36] had developed the Clinical Decision Support System for the diagnostic evaluation of adults and children. This team had proposed the term 'tele-enabling model' for their novel service delivery model, which enabled primary care physicians to diagnose and manage psychiatric problems using detailed decision-making algorithms, through video conferencing with tertiary care specialists (including a child psychiatrist) as and when required.

The pandemic has led to the expansion of telepsychiatry services in government and private medical institutes. 
Furthermore, these services are also being used for providing psychiatric care to people in quarantine and those infected with COVID-19.

\section{Telepsychiatry Guidelines in India}

The Indian Psychiatric Society and Telemedicine Society of India, in collaboration with NIMHANS, had published Telepsychiatry Operational Guidelines in May 2020 [37]. The telemedicine guidelines provide general guidance on prescribing, while the Telepsychiatry Operational Guidelines provide the specific list of medications.

According to the guidelines, parental consent is not essential for an individual above 16 years [12]. However, it is prudent to obtain permission from the parent for anyone below 18 years, given the existing definition of 'minor' in the Mental Health Care Act, 2017 [38]. Several children and adolescents require psychotropic medication alongside psychosocial interventions to manage their behavioural and emotional problems adequately. As per guidelines, barring methylphenidate, which comes under schedule X class of drugs, other psychotropic medications can be prescribed to children through a teleconsultation by a qualified psychiatrist. However, the 'List A' medications that can be prescribed during the first teleconsultation are limited. For example, among the antidepressants, escitalopram and fluoxetine may be prescribed during the initial consultation. However, sertraline, an extremely useful beneficial drug especially for paediatric mood and anxiety disorders, is a 'List B' medication that can only be prescribed during a follow-up consultation. In the initial few weeks of starting medication, children/adolescents need to be monitored for specific side effects that may become particularly problematic for sensitive individuals in this population. In particular, the side effects such as behavioural activation with selective serotonin reuptake inhibitors, extrapyramidal side effects with antipsychotics, and blood pressure changes with clonidine should be watched. This is not possible over telemedicine; thus, a liaison with an accessible medical setup must be ensured for timely monitoring.

\section{Telepsychotherapy for Children and Adolescents}

For psychotherapy, the ethical and legal responsibilities and requirements laid down by the Indian telemedicine guidelines are similar to those for face-to-face therapy. A psychiatrist is entitled to provide teletherapy to patients from any part of India. The guidelines advise a detailed in-person face-to-face assessment before initiating teletherapy. All communications between psychiatrist and patient shall be documented and filed in the patients' medical record, similar to traditional record-keeping policies and procedures. The psychiatrist can choose not to proceed with a teletherapy consultation at any time and, at any point, may feel free to advise an in-person therapy consultation.

\section{Clinical Telemedicine Services at Department of Child and Adolescent Psychiatry, NIMHANS, Bengaluru}

The clinical team at the Department of Child and Adolescent Psychiatry at NIMHANS was able to successfully initiate and sustain telepsychiatry services after the onset of COVID-19, although this was not the norm previously. Like other central government institutes in India, telemedicine services at NIMHANS have not been enabled for clients who are not previously registered with the institute. Thus, only 'old' patients or those who have previously consulted at least once in person were taken up for follow-up teleconsultations. Between April 2020 and March 2021, 2993 children and adolescents were seen over teleconsultation. As clients were initially unaware that teleconsultations were now possible, we had proactively reached out to families who were due for routine follow-up, obtained consent for a teleconsultation, and conducted the same. Children and adolescents who had recently been admitted as in-patients with severe psychiatric problems were identified and contacted on priority to ensure remission from their illness and relapse prevention. Subsequently, a centralized IVRS phone line was set up so that children and families could reach out and request a follow-up teleconsultation according to their convenience. Following the telemedicine guidelines, e-prescriptions were issued and scanned and sent to families.

In keeping with our ethos of working as a multidisciplinary team, psychotherapeutic services and family interventions were also offered and conducted successfully over the telemedicine route. Most children and their families found telemedicine acceptable, effective, and convenient. We did face difficulties in assessing and treating younger children. Observations of activity and behaviour were difficult, and hyperactive toddlers would often go out of the frame. The engagement was also difficult with toddlers and younger children because it is common during in-person practice for the child psychiatrist to move along with the child, trying to engage the child. This is especially so while evaluating children with developmental disabilities such as intellectual disability and autism spectrum disorder. Thus, for preschoolers and children with developmental disabilities, we worked extensively with parents instead of direct behavioural or play therapy with children. Interventions that were successfully delivered included functional behavioural analysis for management of behavioural problems and 
training parents to coach their children to develop new skills in different developmental domains. For older children and adolescents, especially those above the age of 10 years, individual psychotherapy online sessions were attempted as and when deemed feasible. Most adolescents seamlessly transitioned from in-person to online psychotherapy, especially as they already had established a rapport in person previously with their therapist. Some adolescents, especially those with social anxiety disorder felt more comfortable with the distance and 'remoteness' that telepsychotherapy provided and were able to speak more freely than when they would come for sessions to the outpatient clinic. Adolescents with externalizing disorders like attention deficit hyperactivity disorder (ADHD) were more distractible and difficult to engage in online therapy, and especially those with comorbid technology addiction would sometimes be found to have another screen open at the same time as a therapy session. This was incidentally a complaint that their parents would also endorse that during online classes, these youngsters would be engaged with another online game or application at the same time. There were certain situations when telepsychotherapy was deemed unsuitable, and the family was requested either to come to the OPD or consult a mental health practitioner locally. Some of these were when a child or adolescent had come into conflict with the law and required an assessment or if a sensitive issue like child sexual abuse was suspected, which according to Indian law needs to be mandatorily reported to child welfare/law enforcement agencies. A suicidal or aggressive child or adolescent was also immediately asked to report in person to emergency services. Additionally, families with high conflict levels, children who needed trauma-focused psychotherapy, and children who did not seem to be improving adequately with telemedicine were encouraged to come in person to the outpatient department.

\section{Telepsychiatry of the Future}

Virtual reality adaptable devices and technology are already making inroads as supplementary treatment modalities in various psychiatric conditions like phobias, anxiety disorders, depressive disorders, ADHD, and even autistic spectrum disorders. These technologies could be integrated with telepsychiatry for enhanced psychiatric care delivery [39]. Artificial intelligence and machine learning utilize big data collected from various sources, including medical records, wearables, and clients' monitoring devices, and analyse the data using algorithms. This information could revolutionize the practice of telepsychiatry in providing precise and personalized psychiatric care to clients [40].

\section{Teleyoga for Parents, Children, and Adolescents}

The Department of Integrative Medicine, NIMHANS, Bengaluru, has been offering teleyoga services for managing stress levels of parents, children, and adolescents for over a year now. Initially, the department developed a 45-min teleyoga program for stress management and the well-being of adults [41]. Google survey among attendees revealed that morning slot of 7:00 AM to 7:45 AM was the most feasible time to impart these sessions. This program was found feasible and helpful in reducing psychological stress [41]. NIMHANS also developed teleyoga programs for the well-being of children and adolescents. Based on the feasibility, ability to learn yoga, and the level of complexity in yoga practices, two separate yoga modules were developed for children aged 5-10 years and 10-15 years, respectively. The modules included different yoga practices ranging from yogic games (krida yoga), physical postures (asanas), and breathing techniques (pranayama) to meditations. The module for children from 5 to 10 years of age was $30 \mathrm{~min}$ in duration and focused more on asanas, mantra chanting, and yogic games with a small component of pranayama such as right and left nostril breathing and humming breath. The YouTube link of the teleyoga module for children between 5 and 10 years of age is https:// youtu.be/SFZTCIfAm1A? $\mathrm{t}=2$. In the module for children between 10 and 15 years of age, sun salutation practice was added (6-12 rounds), and the asanas were maintained for a longer time (15-30 s). This yoga module was of $50 \mathrm{~min}$ in duration and involved fast- and slow-breathing techniques and meditation and relaxation techniques. The YouTube link for this module is https://youtu.be/pp9Rg CyiMe8? $\mathrm{t}=2$.

During the feasibility study, the teleyoga sessions were conducted six days/week between 10:00 AM and 11:00 AM. The children and adolescents who participated in the sessions were evaluated on Strengths and Difficulty Questionnaire at the baseline and after 1 month. Parental consent and assent from the children were obtained. At the end of 1 month, yoga performance assessment revealed that children and adolescents were able to learn the whole set of yoga practices through tele-mode. The preliminary data on testing the feasibility of these modules revealed significant reduction in the total difficulty scores on the Strengths and Difficulties Questionnaire in children and adolescents. Children showed significant improvements in hyperactivity, peer problems, and prosocial behaviour sub-domains. In the adolescent group, significant improvements were reflected in the sub-domains of hyperactivity, emotional problems, and conduct issues [42]. The parents reported no adverse effects. Most of the parents (90\%) 
have opined that yoga should be included in school curriculum for the enhancement of physical, mental, and emotional well-being of the students. Thus, given the efficacy of teleyoga, we propose that this can be a way to inculcate the practice of yoga among children even during online schooling in the current scenario. However, more extensive clinical trials with robust methodologies are encouraged to test the teleyoga modules that have been developed for both age groups.

\section{Conclusion}

The pandemic has accelerated the growth of telemedicine in India. In a resource-limited country with a vast child and adolescent population and difficult terrain, telemedicine has facilitated 'health for all'. This model of paediatric and adolescent telehealth in India can be replicated by other LMIC. This telemedicine model can be permanently integrated into the healthcare delivery system of India, even after the pandemic ends. Medical education, triaging, referrals, health promotion, and follow-up of paediatric and adolescent medical and mental disorders can be successfully conducted through the tele-mode by trained health professionals. Modifications of the current guidelines, enhanced digital awareness of the population, and technological advances would help to overcome the shortcomings of illiteracy, breach of data privacy, confidentiality, and poor internet connectivity. Telemedicine is here to stay and, in the coming years, would prove to be a game-changer in the field of medical care and education to ensure universal health coverage.

\section{Declarations}

Human and Animal Rights and Informed Consent All reported studies/ experiments with human or animal subjects performed by the authors have been previously published and complied with all applicable ethical standards.

\section{References}

Papers of particular interest, published recently, have been highlighted as:

- Of importance

$\bullet$ Of major importance

1. India: WHO Coronavirus Disease (COVID-19) Dashboard [Internet]. Geneva: WHO. Available from: https://covid19. who. int/region/searo/country/in. Accessed on 26 Aug 2021.

2.• Agarwal N, Jain P, Pathak R, Gupta R. Telemedicine in India: a tool for transforming health care in the era of COVID-19 pandemic. J Educ Health Promot. 2020;28:190. https://doi.org/10. 4103/jehp.jehp_472_20. A comprehensive review of evolution of telemedicine in India.

3. India: https://datareportal.com/reports/digital-2021-india. Accessed on 2 Sep 2021.

4.•• Dash S, Aarthy R, Mohan V. Telemedicine during COVID-19 in India-a new policy and its challenges. J Public Health Policy. 2021;19:1-9. https://doi.org/10.1057/s41271-021-00287-w. An interesting review on India's current and future telemedicine policy.

5. Dhingra D, Dabas A. Global strategy on digital health. Indian Pediatr. 2020;57:356-8. A detailed discussion on WHO's latest digital health policy.

6. Deodhar J. Telemedicine by email-experience in neonatal care at a primary care facility in rural India. J Telemed Telecare. 2002;8:20-1. https://doi.org/10.1177/1357633X020080S209.

7. Chellaiyan VG, Nirupama AY, Taneja N. Telemedicine in India: where do we stand? J Family Med Prim Care. 2019;8:1872-6. https://doi.org/10.4103/jfmpc.jfmpc_264_19.

8. Singh M, Das RR. Four years of experience of telemedicine for paediatric care in three Punjab hospitals, North India: achievements and lessons. Postgrad Med J. 2010;86:688-91. https://doi. org/10.1136/pgmj.2009.082735.

9. Carmichael SL, Mehta K, Srikantiah S, Mahapatra T, Chaudhuri I, Balakrishnan R et al; Ananya Study Group*. Use of mobile technology by frontline health workers to promote reproductive, maternal, newborn and child health and nutrition: a cluster randomized controlled Trial in Bihar, India. J Glob Health. 2019;9:0204249.https://doi.org/10.7189/jogh.09.020424.

10. Chawla D, Thukral A, Kumar P, Deorari A. Harnessing mobile technology to deliver evidence-based maternal-infant care. Semin Fetal Neonatal Med. 2021;26:101206. https://doi.org/ 10.1016/j.siny.2021.101206.

11. Mahajan V, Singh T, Azad C. using telemedicine during the COVID-19 pandemic. Indian Pediatr. 2020;57:652-7.

12. Ministry of Health and Family welfare, Government of India. Telemedicine practice guidelines enabling registered medical practitioners to provide healthcare using tele-medicine. New Delhi. Available at https://www.mohfw.gov.in/pdf/Telemedici ne.pdf. Accessed on 2 Sep 2021.

13. Ray A, Sharma S, Sadasivam B. Telephonic triage in times of COVID-19: experiences at a telemedicine center in India. Disaster Med Public Health Prep. 2021;1-2. https://doi.org/10.1017/ dmp.2021.126.

14. Pataki C, Singhal P, Dastidar AG. UK-India Telemedicine Project \& COVID-19. QJM. 2021; hcab199. https://doi.org/10.1093/ qjmed/hcab199.

15. Mittal A, Pareek P. Telephonic triage and telemedicine during the peak of COVID-19 pandemic - restricting exposure to healthcare professionals. Indian Pediatr. 2020;57:973-4. https://doi. org/10.1007/s13312-020-2011-7.

16. Vinekar A, Mangalesh S, Jayadev C, Gilbert C, Dogra M, Shetty B. Impact of expansion of telemedicine screening for retinopathy of prematurity in India. Indian J Ophthalmol. 2017;65:390-5. https://doi.org/10.4103/ijo.IJO_211_17.

17. Bhaskaran K, Sharma P. Distancing? But still I-care: teleophthalmology during COVID-19 era. Indian J Ophthalmol. 2020;68:1243-4. https://doi.org/10.4103/ijo.IJO_1875_20.

18. Manglani M, Gabhale Y, Lala MM, Balakrishnan S, Bhuyan K, Rewari BB, Setia MS. Reaching the unreached: providing quality care to HIV-infected children through telemedicine-an innovative pilot initiative from Maharashtra. India Int J Pediatr. 2020;2020:6432476. https://doi.org/10.1155/2020/6432476.

19. Manglani M, Gabhale Y, Lala MM, Balakrishnan S, Bhuyan K, Rewari BB, Setia MS. Assessing the effectiveness of a telemedicine initiative in clinical management of children living with HIV/AIDS in Maharashtra. India Curr HIV Res. 
2021;19:201-15. https://doi.org/10.2174/157339981766621 0104102825 .

20. Pandey V, Srivastava V, Imran M, Mishra A. Active telephonic follow-up during COVID-19 lockdown: initial experience. Indian Pediatr. 2020;57:769. https://doi.org/10.1007/ s13312-020-1937-0.

21. Banothu KK, Bhat JI, Das RR, Dhochak N, Ghimire JJ, Goyal JP, et al. Expert opinion on restoration of pediatric pulmonology services during the SARS-CoV-2 pandemic. Indian Pediatr. 2020;57:1055-9. https://doi.org/10.1007/s13312-020-2035-z.

22. Kumari J, Jat KR, Kabra SK. Role of telemedicine in followup care of children with respiratory illnesses at a tertiary care hospital - an ambispective observational study. Indian J Pediatr. 2021;1-5. https://doi.org/10.1007/s12098-020-03590-8.

23. Lahiri D, Mitra S. COVID-19 is accelerating the acceptance of telemedicine in India. J Family Med Prim Care. 2020;9:3785-6. https://doi.org/10.4103/jfmpc.jfmpc_580_20.

24. Sharawat IK, Panda PK. Caregiver satisfaction and effectiveness of teleconsultation in children and adolescents with migraine during the ongoing COVID-19 pandemic. J Child Neurol. 2021;36:296-303. https://doi.org/10.1177/0883073820968653.

25. Panda PK, Dawman L, Panda P, Sharawat IK. Feasibility and effectiveness of teleconsultation in children with epilepsy amidst the ongoing COVID-19 pandemic in a resource-limited country. Seizure. 2020;81:29-35. https://doi.org/10.1016/j.seizure.2020. 07.013 .

26 Saini L, Madaan P, Bhagwat C, Einspieler C. Home-videos for neurodevelopmental follow-up of high-risk infants during COVID-19 pandemic: a simple and inexpensive tool. J Trop Pediatr. 2021;67(1):fmaa088. https://doi.org/10.1093/tropej/ fmaa088.

27. Ganguly S, Bakhshi S. Teleconsultations and shared care in pediatric oncology during COVID-19. Indian J Pediatr. 2021;88:1-2. https://doi.org/10.1007/s12098-020-03499-2.

28. Gulati S, Sengar A. Experience with telemedicine in paediatric nephrology during the COVID pandemic. Pediatr Nephrol. 2021;36:2499-500. https://doi.org/10.1007/ s00467-021-05085-w.

29. Menon J, Kannankulangara A. Providing medical services online to children with chronic kidney disease during the COVID-19 pandemic. Indian Pediatr. 2021;58:291. https://doi.org/10.1007/ s13312-021-2181-y.

30. Muthukrishnan J, Venugopal N, Basavaraj AP, Bagga G, Jayakrishnan VY, Bharadwaj K, Thoke SV. Management of type 1 diabetes mellitus during the COVID-19 pandemic. Med J Armed Forces India. 2021;77:S393-7. https://doi.org/10.1016/j.mjafi. 2021.04.001.

31. Devassy SM, Allagh KP, Benny AM, Scaria L, Cheguvera N, Sunirose IP. Resiliency Engagement and Care in Health
$(\mathrm{REaCH})$ : a telephone befriending intervention for upskilled rural youth in the context of COVID-19 pandemic-study protocol for a multi-centre cluster randomised controlled trial. Trials. 2021;22:500. https://doi.org/10.1186/s13063-021-05465-5.

32. Chandrasekaran S, Chandrashekar VS, Dalvie S, Sinha A. The case for the use of telehealth for abortion in India. Sex Reprod Health Matters. 2021;29:1920566. https://doi.org/10.1080/26410 397.2021.1920566.

33. Allely EB. Synchronous and asynchronous telemedicine. J Med Syst. 1995;19:207-12.

34. Manjunatha N, Kumar CN, Math SB. Coronavirus disease 2019 pandemic: time to optimize the potential of Telepsychiatry aftercare clinic to ensure the continuity of care. Indian J Psychiatry. 2020;62:320-1.

35. Thara R, John S, Rao K. Telepsychiatry in Chennai, India: the SCARF experience. Behav Sci Law. 2008;26:315-22.

36. Malhotra S, Shah R. Telepsychiatry and digital mental health care in child and adolescent psychiatry. In Understanding uniqueness and diversity in child and adolescent mental health. Elsevier Inc. 2018. https://doi.org/10.1016/b978-0-12-815310-9. 00011-3.

37. Math SB, Manjunatha N, Kumar CN, et al. Telepsychiatry operational guidelines. Bengaluru: NIMHANS, 2020. Available at:https://indianpsychiatricsociety.org/e-book-Telepsychi atry-operational-guidelines-2020. Accessed on 2 Sep 2021.

38. The Mental Health Care Act 2017. Available at: https://www. indiacode.nic.in/bitstream/123456789/2249/1/a2017-10.pdf.

39. Vadlamani LN, Sharma V, Emani A, Gowda MR. Telepsychiatry and outpatient department services. Indian J Psychol Med. 2020;42:27S-33S. https://doi.org/10.1177/0253717620960694.

40. Koppe G, Meyer-Lindenberg A, Durstewitz D. Deep learning for small and big data in psychiatry. Neuropsychopharmacology. 2020. https://doi.org/10.1038/s41386-020-0767-z.

41. Jasti N, Bhargav H, George S, Varambally S, Gangadhar BN. Tele-yoga for stress management: Need of the hour during the COVID-19 pandemic and beyond? Asian J Psychiatry. 2020;54:102334.

42. Abraham SE, Jasti N, Bhargav H, Galagali PM, Varambally S. Design, validation and feasibility of a tele yoga module for adolescents well-being. Indian Pediatrics, Manuscript submitted, 2021.

Publisher's Note Springer Nature remains neutral with regard to jurisdictional claims in published maps and institutional affiliations. 